\title{
An effective patient training for deep inspiration breath hold technique of left-sided breast on computed tomography simulation procedure at King Chulalongkorn Memorial Hospital
}

\author{
Puntiwa Oonsiri', Metinee Wisetrinthong ${ }^{1}$, Manatchanok Chitnok1, Kitwadee Saksornchai', Sivalee Suriyapee ${ }^{2}$ \\ 'Division of Radiation Oncology, King Chulalongkorn Memorial Hospital, The Thai Red Cross Society, Bangkok, Thailand \\ ${ }^{2}$ Division of Radiation Oncology, Department of Radiology, Faculty of Medicine, Chulalongkorn University, Bangkok, Thailand
}

\begin{abstract}
Purpose: To observe the effectiveness of the practical instruction sheet and the educational video for left-sided breast treatment in a patient receiving deep inspiration breath hold (DIBH) technique. Two parameters, simulation time and patient satisfaction, were assessed through the questionnaire.

Methods: Two different approaches, which were the instruction sheet and educational video, were combinedly used to assist patients during DIBH procedures. The guideline was assigned at least 1 week before the simulation date. On the simulation day, patients would fill the questionnaire regarding their satisfaction with the DIBH instruction. The questionnaire was categorized into five levels: extremely satisfied to dissatisfied, sequentially. The patients were divided into four groups: not DIBH technique, DIBH without instruction materials, the DIBH with instruction sheet or educational video, and DIBH with both of instruction sheet and educational video.

Results: Total number of 112 cases of left-sided breast cancer were analyzed. The simulation time during DIBH procedure significantly reduced when patients followed the instruction. There was no significant difference in simulation time on the DIBH procedures between patient compliance via instruction sheet or educational video or even following both of them. The excellent level was found at $4.6 \pm 0.1$ and $4.5 \pm 0.1$, for patients coaching via instruction sheet as well as on the educational video, respectively.

Conclusion: Patient coaching before simulation could potentially reduce the lengthy time in the simulation process for DIBH technique. Practicing the DIBH technique before treatment is strongly advised.
\end{abstract}

Keywords: Deep inspiration breath-hold (DIBH), Left-sided, Breast cancer, Cardiac toxicity

\section{Introduction}

Post-operative radiation therapy is the common treatment of breast cancer, which can reduce the locoregional recurrent rate. Breast cancer is a common challenge in radiotherapy due to a variety of treatment conditions. Many techniques are applied, such as intensity-modulated radiation therapy or volumetric modulated radiation therapy used for bilateral

Received 22 May 2019, Revised 22 June 2019, Accepted 01 July 2019.

Correspondence: Puntiwa Oonsiri, Division of Radiation Oncology, Department of Radiology, King Chulalongkorn Memorial Hospital, 1873 Rama IV Road, Pathumwan, Bangkok 10330, Thailand. Tel: +66-02-256-4334, E-mail: nim_1000d@hotmail.com (http://orcid.org/0000-0002-0226-3723)

(c) This is an Open Access article distributed under the terms of the Creative Commons Attribution Non-Commercial License (http://creativecommons.org/ licenses/by-nc/4.0/) which permits unrestricted non-commercial use, distribution, and reproduction in any medium, provided the original work is properly cited.

www.e-roj.org 


\section{Puntiwa Oonsiri, et al}

breast irradiation [1], breathing controlled used for left side breast irradiation. However, breast irradiation treatment usually consists of medial and tangential beams designed to reduce radiation dose to the underlying heart and healthy lung tissue [2]. Patient immobilization in conjunction with treatment planning is essential to reduce the irradiated volume of the healthy tissues. Especially for the left breast irradiation, a low dose of radiation may induce the late complication to the heart such as the morbidity and mortality, even though the complication may also arise from other factors, such as systemic treatment and patient's comorbidities [3]. Many studies reported the radiation dose to the heart on left breast irradiation [4-6].

One of the advanced treatment techniques which are more sophisticated to reduce the radiation dose to the heart is deep inspiration breath hold (DIBH) technique. Several of the literature confirmed that this technique could reduce the radiation dose to the heart [5-14] and lungs $[10,13,15-18]$ during left beast irradiation. For the DIBH technique, patients should take deep inspiration and hold for approximately 20 seconds during the simulation or treatment process. The air filling inside the chest will move the heart position away from the treatment field. This technique will gain success once the patient completes the breath hold compliance. Motion management training is essential preparation for this group of patient. Our center has started treating patients with DIBH technique since 2015. The tools that used to track the breath hold position during the simulation process was the RPM marker block while a 3D surface imaging system (AlignRT; Vision RT Ltd., London, UK) was used to track the real-time patient position in treatment delivery.

The main problem which deteriorates the success of DIBH procedure is the patient's pressure during the simulation. The training session takes around 20-30 minutes, which is tedious yet making patients stress and uncomfortable. Although radiation oncologists have explained to patients about the information on DIBH technique before the treatment, defects still probably occur and generate a serious problem, for example, the unclear detail in the patient's body. We initiated the idea to create an information sheet, so patients can learn the DIBH in detail and train themselves before the simulation starts. Not only the instruction sheet but also the tutorial video that depicts the process of simulation for DIBH patient were created. This document was handed to the patient one week before the simulation session. For the video, it can be easily accessed via our department website (http://www.chulacancer. net), QR code scanning, or browsing via Google or Youtube.
Radiation Oncology Journal ROJ

This article reviewed the success of implementing the instruction sheet and educational video for patients who underwent radiation treatment with DIBH technique. The feasibility and efficacy of the guidelines were evaluated based on the simulation time, started from a patient lying down on the couch until image acquisition was completed. The laborious time during computed tomography (CT) simulation process declined when patients obeyed the practical guidelines.

\section{Materials and Methods}

The patients with left breast cancer who underwent DIBH procedure from October 2017 to August 2018 were observed. The patients were divided into four groups: group $A$ is the patients who did not treat with DIBH technique, group $B$ is the DIBH patients without instruction material (before the instruction sheet and educational video launched), group C is the DIBH patients with instruction material via instruction sheet or educational video, and group D is the DIBH patients with instruction material for both sheet and video. The group $\mathrm{C}$ and $\mathrm{D}$ patients, the data collected after the instruction sheet and educational video were launched. To avoid unwanted bias results, the patients of group $C$ and $D$ were not assigned or suggested any instruction materials for them, but they can select the preferable methods. The selected materials from patients were confirmed again via remarkable items on the questionnaire.

The information sheet comprised of the aim of DIBH technique, step by step for practice, figures, and the QR code to access the 3-minute educational video, where patients can directly follow its instructions. This guideline was given at least 1 week before the simulation started. Both Thai and English languages were provided. Fig. 1 shows the printed document on both sides on size A5 ( $5.8 \times 8.3$ inches).

On the simulation date, patients must fill up the questionnaire regarding their satisfaction of both document and video before the CT simulation started. The questionnaire consists of two parts. The first part composes of the personal history of the patient, such as age, patient's education group the experience of radiotherapy, and the information on the DIBH technique. The second part is the level of satisfaction, which categorized into five levels: extremely satisfied, very satisfied, moderately satisfied, dissatisfied, and extremely dissatisfied. Those levels were represented by score 5, 4, 3, 2, and 1 for each level, respectively. We explained to patients that the questionnaire did not influence the treatment process.

The Philips Brilliance Big Bore 16-slice and Siemens 

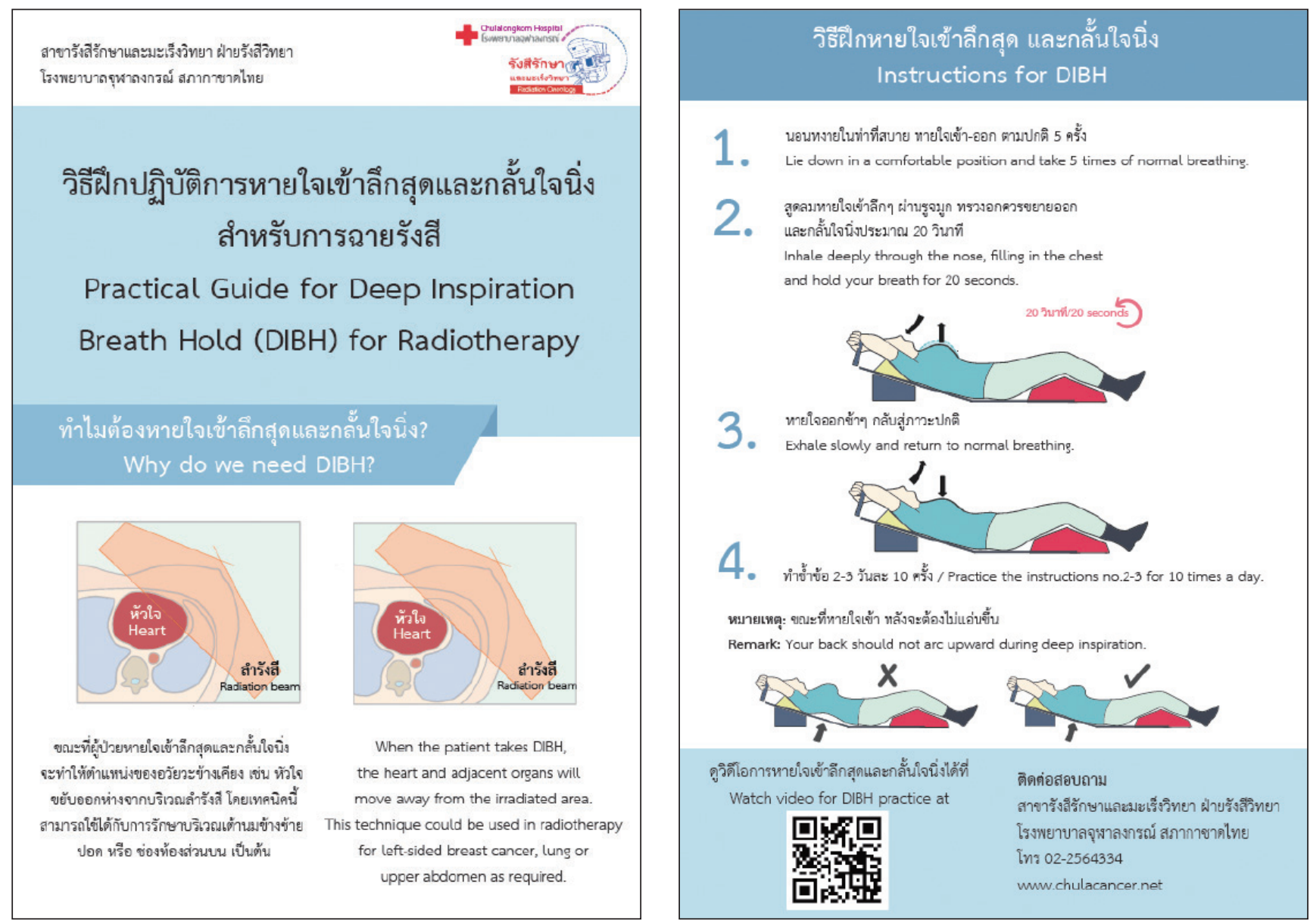

Fig. 1. The practical guide for DIBH document with a $Q R$ code at the end of the instruction.

SOMATOM Definition AS 64-slice CT simulation were utilized in our department. The patient position was supine, and both hands were raised overhead. The breast board (CIVCO Medical Solutions, Orange City, IA, USA) was used for immobilization. The Varian real-time position management (RPM, Varian Medical System, Palo Alto, CA, USA) observed the breathhold level from the respiratory curve at CT simulation. The simulation time process was observed from patient positioning until completion of CT scanning. The images data set was acquired in the free breath and DIBH technique for all cases. The range of scanning covered the ears to $L 2$ with $3 \mathrm{~mm}$ of slice thickness.

\section{Results}

The total of 112 cases was analyzed; $92.6 \%$ of these patients never had an experience in radiotherapy before. There was no significant difference among the average age of patients for all groups as depicted in Table 1. The simulation time for DIBH cases which was categorized into four groups is listed in Table 1.

The simulation time reduced significantly when the patient followed the practical guidelines. However, the difference was not significant between the DIBH with instruction materials via sheet or video or using both of them. It could imply that either the instruction sheet or video was enough depending on the patient's preference.

The satisfaction scoring parameters of the questionnaire were evaluated for the practical guide document and the video as can be seen in Table 2. All parameters showed an excellent level, $4.6 \pm 0.1$ and $4.5 \pm 0.1$ for document and video, respectively.

The coaching strategy for DIBH patients is essential. Many factors are affecting the success in the process-for instance, age, voluntary, the condition of patients, etc. [19]. This study did not limit the patient's age for the DIBH technique. However, radiation oncologist observed patient's status by physical observation such as the patient who can cooperate in the $\mathrm{DIBH}$ training or who do not have any respiratory function 
Table 1. Simulation time of DIBH techniques according to the patient groups

\begin{tabular}{lcccc}
\hline \multicolumn{1}{c}{ Category } & Number of cases & Age $(\mathrm{yr})$ & Duration of simulation (min) & $\mathrm{p}$-value \\
\hline Not DIBH technique & 25 & $52.6 \pm 11.2$ & $8.0 \pm 2.9$ & $22.3 \pm 8.0$ \\
DIBH without instruction materials & 23 & $49.4 \pm 10.4$ & $10.3 \pm 2.8$ & $0.1^{*}$ \\
DIBH with patient instruction via sheet or video & 30 & $49.5 \pm 10.1$ & 0.10 & $9.7 \pm 2.7$ \\
DIBH with patient instruction via sheet and video & 34 & $51.4 \pm 11.5$ & 0.17 \\
\hline
\end{tabular}

Values are presented as mean \pm standard deviation.

DIBH, deep inspiration breath hold.

*By two-tail Student t-test.

Table 2. Satisfaction scoring parameters from the practical guide document and the video

\begin{tabular}{|c|c|c|}
\hline Topic & Document (score) & Video (score) \\
\hline Issue (substance) completely & 4.5 & 4.5 \\
\hline Clarify content & 4.6 & 4.5 \\
\hline Illustration & 4.6 & 4.6 \\
\hline Induce to do practice & 4.5 & 4.5 \\
\hline Average & $4.6 \pm 0.1$ & $4.5 \pm 0.1$ \\
\hline
\end{tabular}

Values are presented as mean \pm standard deviation.

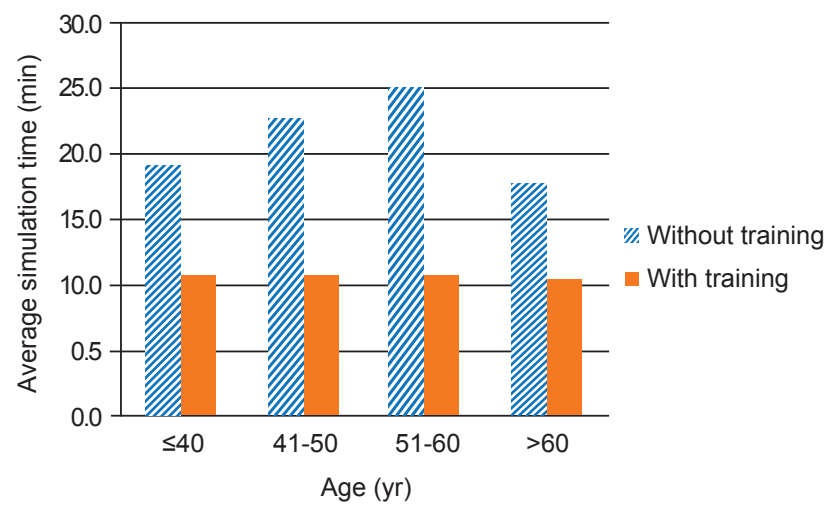

Fig. 2. Relationship between patient's age and simulation time with and without training.

impairment. Relationship between patient's age and simulation time was explored in Fig. 2. The average simulation time was reduced significantly after training for all range of the age. Nevertheless, there was no significant difference in simulation time among the group of age after training. This result indicated that the instruction sheet and educational video for DIBH training could be employed within all range of patient's age. The threshold point of patient's age for non-beneficial of DIBH is still unambiguous in this study. Czeremszynska et al. [19] supported that the age alone cannot be appointed as a criteria for the DIBH technique.

The average simulation time and patient's education relationship was shown in Fig. 3. For each patient's education

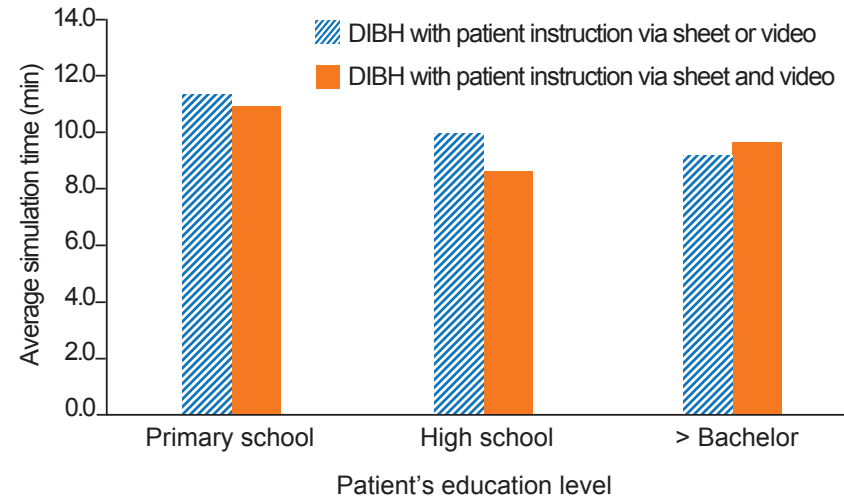

Fig. 3. Average simulation time for different groups based on patient's education. DIBH, deep inspiration breath hold.

group, there was no significant difference in the average simulation time $(p>0.05)$ for patients who used the instruction sheet or video alone, or used both of them for all groups. The simulation time was comparable between the group of the patient's education with no significantly different $(p>0.05)$.

The treatment time of DIBH training is out of the scope of this study since different modality was used in breath hold monitor. The real-time 3D surface imaging system (AlignRT) equipped on the linear accelerator was utilized for setup verification in the delivery process while the Varian real-time position management (RPM) was used in CT simulation. 


\section{Discussion and Conclusion}

The instruction materials confirmed its efficacy to save the simulation time. It does not mean save only time itself, but also implicitly increase the cost-effectiveness because the staff workload decreased and was not considered as burden for patients. Decreasing the workload may increase the number of patients on the simulation procedure. In addition, it also could reduce their waiting time.

The patient coaching before simulation could potentially reduce the lengthy time on the simulation process for DIBH technique. The different outcome of patient compliance using either instruction sheet or video or both of them were not significant. This study encourages patients to practice the DIBH technique properly as it can enhance the success of leftsided breast treatment.

\section{Conflict of Interest}

No potential conflict of interest relevant to this article was reported.

\section{Acknowledgments}

This study was supported by Hospital Accredation of King Chulalongkorn Memorial Hospital. The authors would like to express the most sincere gratitude to Mr. Sammuel Mamesa, medical physicist of Gekha Karunia Abadi company, Indonesia, for English approved.

\section{References}

1. Oonsiri P, Saksornchai K, Suriyapee S. Plan evaluation of intensity modulated radiation therapy and volumetric modulated arc therapy in bilateral breast irradiation with 3-isocenter technique. J Assoc Med Sci 2018;51:81-4.

2. Stefanovski Z, Smichkoska S, Petrova D, Lazarova E.. Advantages of the technique with segmented field for tangential breast irradiation. Proceedings of the $3 \mathrm{rd}$ Conference on Medical Physics and Biomedical Engineering; 2013 Oct 18-19; Skopje, Macedonia. p. 75-8.

3. Bird BR, Swain SM. Cardiac toxicity in breast cancer survivors: review of potential cardiac problems. Clin Cancer Res 2008;14:14-24.

4. Clarke M, Collins R, Darby $S$, et al. Effects of radiotherapy and of differences in the extent of surgery for early breast cancer on local recurrence and 15-year survival: an overview of the randomised trials. Lancet 2005:366:2087-106.

5. Early Breast Cancer Trialists' Collaborative Group. Favourable and unfavourable effects on long-term survival of radiotherapy for early breast cancer: an overview of the randomised trials. Lancet 2000;355:1757-70.

6. Paszat LF, Mackillop WJ, Groome PA, Schulze K, Holowaty E. Mortality from myocardial infarction following postlumpectomy radiotherapy for breast cancer: a populationbased study in Ontario, Canada. Int J Radiat Oncol Biol Phys 1999;43:755-62.

7. Latty D, Stuart KE, Wang W, Ahern V. Review of deep inspiration breath-hold techniques for the treatment of breast cancer. J Med Radiat Sci. 2015;62:74-81.

8. Smyth LM, Knight KA, Aarons YK, Wasiak J. The cardiac dosesparing benefits of deep inspiration breath-hold in left breast irradiation: a systematic review. J Med Radiat Sc. 2015;62:6673.

9. Mast ME, van Kempen-Harteveld L, Heijenbrok MW, et al. Leftsided breast cancer radiotherapy with and without breathhold: does IMRT reduce the cardiac dose even further? Radiother Oncol 2013;108:248-53.

10. Swanson T, Grills IS, Ye H, et al. Six-year experience routinely using moderate deep inspiration breath-hold for the reduction of cardiac dose in left-sided breast irradiation for patients with early-stage or locally advanced breast cancer. Am J Clin Oncol 2013;36:24-30.

11. Osman SO, Hol S, Poortmans PM, Essers M. Volumetric modulated arc therapy and breath-hold in image-guided locoregional left-sided breast irradiation. Radiother Oncol 2014;112:17-22.

12. Remouchamps $V M$, Letts $N$, Vicini $F A$, et al. Initial clinical experience with moderate deep-inspiration breath hold using an active breathing control device in the treatment of patients with left-sided breast cancer using external beam radiation therapy. Int J Radiat Oncol Biol Phys 2003;56:704-15.

13. Remouchamps VM, Vicini FA, Sharpe MB, Kestin LL, Martinez AA, Wong JW. Significant reductions in heart and lung doses using deep inspiration breath hold with active breathing control and intensity-modulated radiation therapy for patients treated with locoregional breast irradiation. Int J Radiat Oncol Biol Phys 2003;55:392-406.

14. Krauss DJ, Kestin LL, Raff G, et al. MRI-based volumetric assessment of cardiac anatomy and dose reduction via active breathing control during irradiation for left-sided breast cancer. Int J Radiat Oncol Biol Phys 2005;61:1243-50.

15. Zurl B, Stranzl H, Winkler P, Kapp KS. Quantitative assessment of irradiated lung volume and lung mass in breast cancer 
Puntiwa Oonsiri, et al

patients treated with tangential fields in combination with deep inspiration breath hold (DIBH). Strahlenther Onkol 2010;186:157-62.

16. Korreman SS, Pedersen AN, Aarup LR, Nottrup TJ, Specht L, Nystrom H. Reduction of cardiac and pulmonary complication probabilities after breathing adapted radiotherapy for breast cancer. Int J Radiat Oncol Biol Phys 2006;65:1375-80.

17. Bruzzaniti $V$, Abate $A$, Pinnaro $P$, et al. Dosimetric and clinical advantages of deep inspiration breath-hold (DIBH) during radiotherapy of breast cancer. J Exp Clin Cancer Res 2013;32:88

18. Nissen HD, Appelt AL. Improved heart, lung and target dose with deep inspiration breath hold in a large clinical series of breast cancer patients. Radiother Onco. 2013;106:28-32.

19. Czeremszynska B, Drozda S, Gorzynski M, Kepka L. Selection of patients with left breast cancer for deep-inspiration breathhold radiotherapy technique: results of a prospective study. Rep Pract Oncol Radiother 2017;22:341-8. 